\title{
Hemoperitoneum Caused by a Spontaneously Ruptured Gastrointestinal Stromal Tumor with Cystic Degeneration
}

\author{
Jin Hyung Lee \\ Department of Surgery, Soonchunhyang University Gumi Hospital, Soonchunhyang University College of Medicine, Gumi, Korea
}

\begin{abstract}
A 39-year-old man visited Soonchunhyang University Gumi Hospital with abdominal pain. Abdominal computed tomography showed a large heterogeneously enhancing mass abutting the stomach body and a large amount of fluid collected in the peritoneal cavity. An emergency laparotomy revealed a ruptured large tumor arising from the anterior wall of the middle section of the stomach with bloody fluid in the peritoneal cavity. The patient underwent wedge resection of the tumor. The tumor was $6.5 \times 5.5 \times 4.0 \mathrm{~cm}$ in size and $49 \mathrm{~g}$ in weight. Immunohistochemical staining demonstrated positivity to C-kit protein, CD34, and S-100 protein. The final diagnosis was gastric gastrointestinal stromal tumor (GIST). Hemoperitoneum caused by a spontaneously ruptured GIST with cystic degeneration is extremely rare.
\end{abstract}

Keywords: Gastrointestinal stromal tumors; Rupture; Hemoperitoneum

\section{INTRODUCTION}

Gastrointestinal stromal tumors (GISTs) were first described in 1983 by Mazur and Clark [1], and have been distinguished immunohistochemically from leiomyomas, leiomyosarcoma, and other mesenchymal tumors. There are typical immunohistochemical makers enabling the diagnosis of GISTs. The major diagnostic criteria are the expression of CD117 antigen, a c-kit proto-oncogene product, and CD34, a human progenitor cell antigen. GISTs are the most common mesenchymal tumor of the gastrointestinal tract. The stomach is the most common site of involvement (around 60\%), followed by the small intestine (around 30\%), the colon and rectum (5\%), and the esophagus (<5\%) [2]. Clinical presentation is usually characterized by gastrointestinal bleeding, abdominal pain, weight loss, and a palpable mass. However, most GISTs are asymptomatic, and often represent an incidental finding at surgery or endoscopic examination $[2,3]$.

We describe a case in which a spontaneous ruptured gastrointestinal tumor was associated with non-traumatic hemoperitoneum.

\section{CASE REPORT}

A 39-year-old man had presented with dull upper abdominal pain of one-day duration. His past history indicated that he was diagnosed with arrhythmia two years ago but did not receive any treatment. He had also undergone an operation for a herniated intervertebral disk two year ago. He had no significant family history. On physical examination, there was localized tenderness in the left upper abdomen but no palpable abdominal mass. The laboratory findings revealed no abnormalities except for a slightly low hemoglobin level of $11.9 \mathrm{~g} / \mathrm{dL}$. An abdominal ultrasound examination showed a mass-like lesion with a cystic component between the lesser curvature of the stomach and the lateral segment of the liver. Initial abdominal computed tomography $(\mathrm{CT})$ revealed a $7 \times$ $4 \mathrm{~cm}$ sized heterogeneous enhancing mass abutting the stomach body as well as fluid collection in the right subphrenic area and the pelvic cavity (Fig. 1). A gastroscopy showed a slightly protruded lesion in the upper body of the stomach with no mucosal abnormalities, and an endoscopic ultrasonography revealed an exophytic
Correspondence to: Jin Hyung Lee

Department of Surgery, Soonchunhyang University Gumi Hospital, Soonchunhyang University College of Medicine,

1791 (ill)gongdan-ro, Gumi 39371, Korea

Tel: +82-54-468-9092, Fax: +82-54-468-9097, E-mail: lepus9475@naver.com

Received: May 4, 2017 / Accepted after revision: May 26, 2017
(C) 2017 Soonchunhyang Medical Research Institute This is an Open Access article distributed under the terms of the Creative Commons Attribution Non-Commercial License (http://creativecommons.org/licenses/by-nc/4.0/). 

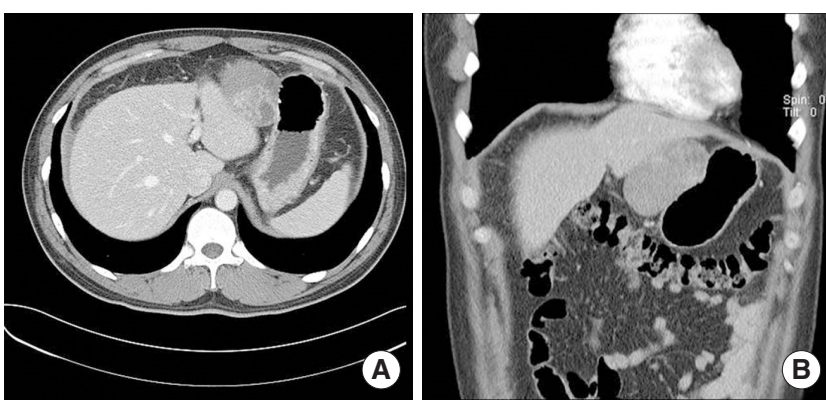

Fig. 1. (A, B) Abdominal computed tomography revealed a heterogeneous enhancing mass abutting the stomach body.
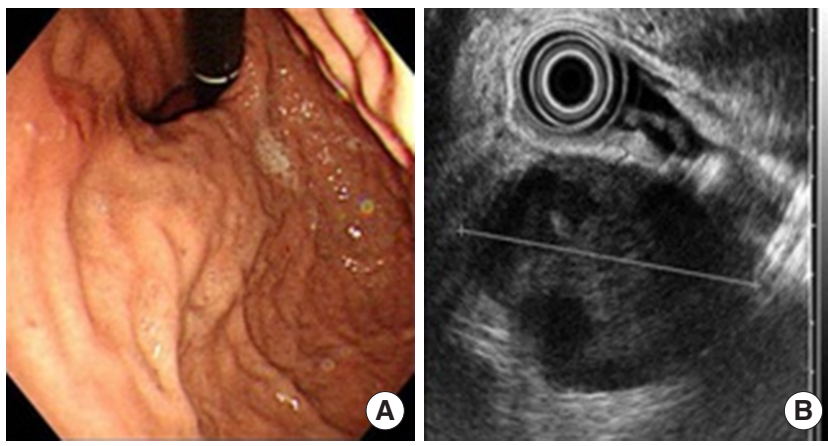

Fig. 2. (A) Gastroscopy showed a slightly protruded lesion. (B) Endoscopic ultrasonography revealed an exophytic mass originating from muscularis propria.

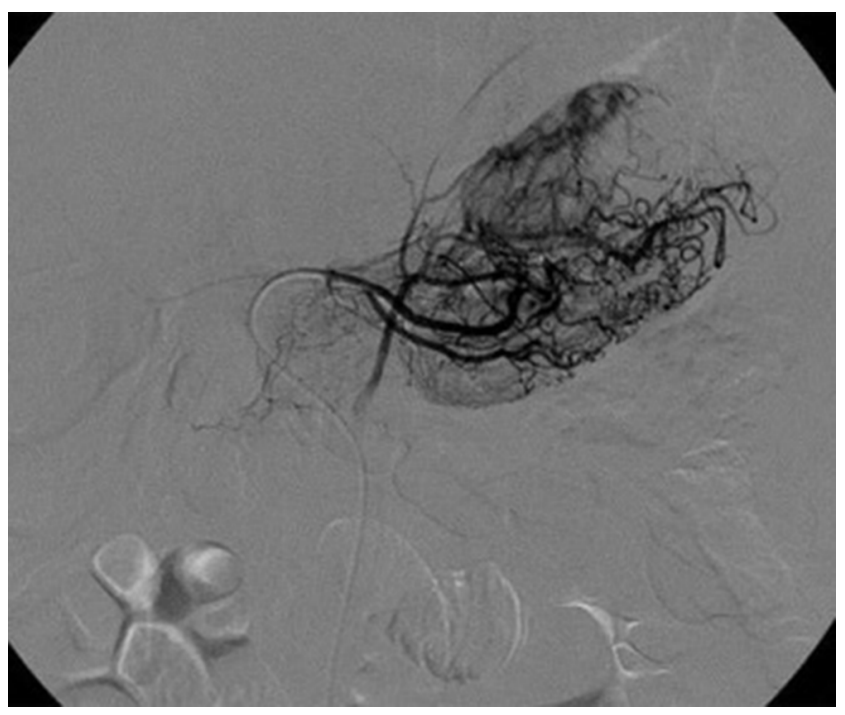

Fig. 3. Abdominal angiography revealed a hypervascular tumor fed by the right gastric artery.

mass originating from the muscularis propria (Fig. 2). Abdominal angiography revealed a hypervascular tumor fed by the right gastric artery. Positron emission tomography CT showed mild hypermetabolism in the large mass adjacent to the stomach (Fig. 3).
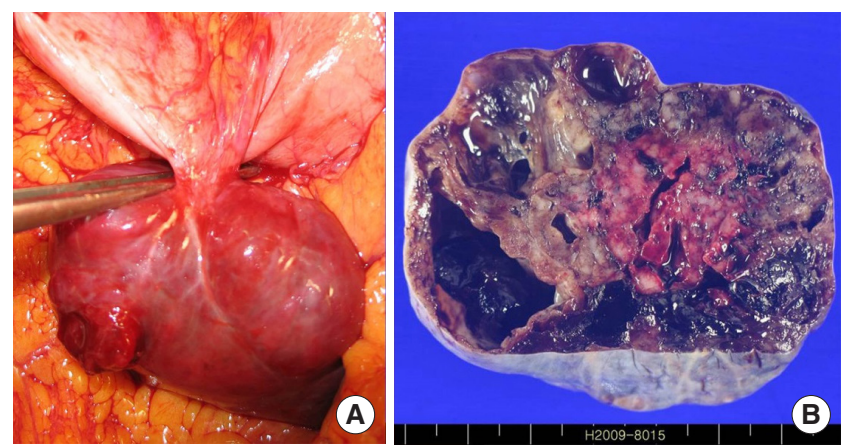

Fig. 4. (A) A ruptured large tumor arising from the anterior wall of the mid-body of the stomach. (B) On sectioning, the resected tumor appeared as brown-tan colored solid mass with areas of cystic degeneration and hemorrhage.
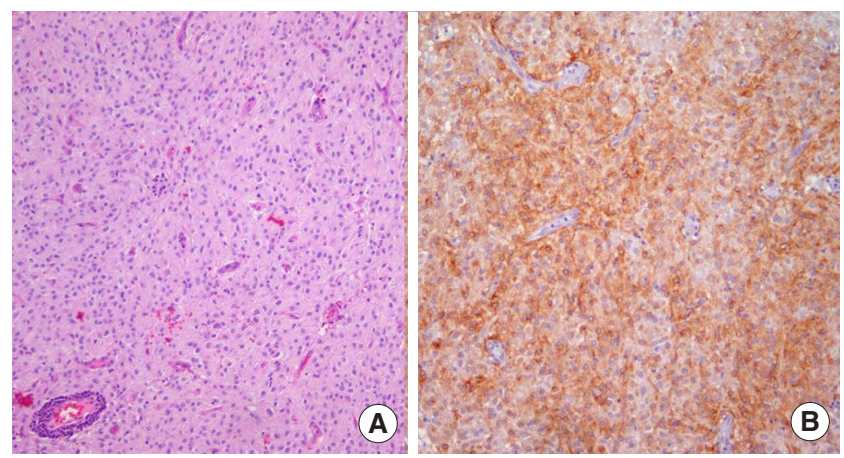

Fig. 5. (A) Microscopically, tumor cells were of the epithelioid type (H\&E, $\times 100$ ). (B) Immunohistochemical staining of the tumor tissue demonstrated as positive result for c-kit $(H \& E, \times 100)$.

Acting on a diagnosis of hemoperitoneum due to the ruptured gastric mass, an emergency laparoscopic exploration was performed. When the intra-abdomen was observed laparoscopically, bloody ascites and a large multicystic tumor arising from the stomach were confirmed. There was immediate conversion to a laparotomy. At laparotomy, the exploration showed a ruptured large tumor arising from the anterior wall of the mid-body of the stomach. The tumor was attached to the gastric wall with a narrow pedicle. The resected tumor was a gray-brown colored ovoid mass, measuring $6.5 \times 5.5 \times 4.0 \mathrm{~cm}$ in size and weighting $49 \mathrm{~g}$ (Fig. 4). On sectioning, it appeared as brown-tan colored solid mass with areas of cystic degeneration and hemorrhage. The solid component showed a whitish tan and lobulating cut surface. Microscopically, tumor cells were of the epithelioid type. Three to five mitotic figures per high power field (HPF) were seen. Immunohistochemical staining of the tumor tissue demonstrated as positive results for c-kit, CD34, S-100 protein, and vimentin, as well as negative results for actin and desmin (Fig. 5). The Ki-67 prolifera- 
Lee JH • A Spontanously Ruptured Gastrointestinal Stromal Tumor

tion index was less than 5\%. The final diagnosis was GIST with low malignant potential. The postoperative course was uneventful and the patient was discharged on the sixth postoperative day. $\mathrm{He}$ was put on imatinib mesylate $400 \mathrm{mg}$ once daily in the outpatient clinic.

\section{DISCUSSION}

There are some studies on the morphology of stomach GISTs. Iwashita [3] also analyzed the macroscopic morphology of 81 cases of GIST and reported that the incidence of the endoluminal type, exoluminal type, intramural type, and mixed types was $35.8 \%$, $33.3 \%, 23.4 \%$, and $7.4 \%$, respectively. Although the exoluminal type is the second most frequent, exophytic pedunculated GISTs originating from the stomach are very rare [3].

The most common symptoms are gastrointestinal bleeding, abdominal pain, weight loss, and a palpable mass. Bleeding into the gastric lumen usually occurs and is caused by tumor ulceration at the mucosal level [4]. However, our case and other reports described massive intraabdominal bleeding caused by spontaneously ruptured gastric GISTs. Most of these cases are characterized by cystic degeneration and a hypervascular tumor. Although the cause of spontaneous rupture of gastric GIST is not known, Cheon et al. [5] suggested that the rupture may be at the weakened wall of the mass, which may be due to cystic degeneration.

The surgical resection of localized gastric GISTs is the preferred treatment modality. Previous consensus was that complete en bloc resection of the tumor with a 1 to $2 \mathrm{~cm}$ is the standard of care for localized GISTs. However De Matteo et al. [6] demonstrated that the status of microscopic margins did not influence the prognosis or the pattern of recurrence. It is therefore accepted that the surgical goal should be a complete resection with gross negative margins only [6]. According to the National Comprehensive Cancer Network's clinical practice guidelines for the optimal management of patients with GIST, laparoscopic techniques should be limited to tumors of less than $2 \mathrm{~cm}$. Recently, several retrospective cohort studies have suggested that laparoscopic resection is feasible and safe for gastric GISTs smaller than $5 \mathrm{~cm}$ and is less invasive than open surgery with similar oncological results [7]. We attempted the laparoscopic technique first. We immediately converted to open surgery because of the tumor's large size (about 8 $\mathrm{cm})$ and spontaneous rupture. However, some case series have reported the successful and safe removal of gastric GISTs of sizes up to $8 \mathrm{~cm}$. There are few case reports of the successful laparoscopic treatment of bleeding GISTs [8].

Although complete excision is the primary treatment modality, almost half of the patients have disease recurrence following surgery, which highlights the need for an effective adjuvant therapy. Imatinib was the first Food and Drug Administration approved agent for metastatic GISTs. In the subsequent SSG (Scandinavian Sarcoma Group and Sarcoma Group of the Arbeitsgemeinschaft Internistische Onkologie [AIO] multicenter) XVIII/AIO phase III randomized controlled trial, 400 patients were randomized based on high-risk features (at least one of following: tumor size $>10 \mathrm{~cm}$, mitotic rate 10/50 HPF, tumor rupture before or during surgery) to either 1 or 3 years of adjuvant imatinib. Based on the result of this trial, three-year adjuvant imatinib therapy is presently the standard of care in the high-risk group [9]. In our case, neither the size nor the mitotic rate would be considered indicative of highrisk; however, adjuvant therapy was administered since the tumor ruptured before surgery.

From the histological point of view, in $45 \%$ of cases the tumor cells were of the spindle type, in 25\% epithelioid, and in 30\% the tumor showed a mixed histotype. In the series analyzed by Singer and colleagues, patients with GISTs having a mixed spindle cell/ epithelioid cell or pure epithelioid cell morphology had poorer 5 -year recurrence free survival than those with pure spindle cell histology [10].

\section{REFERENCES}

1. Mazur MT, Clark HB. Gastric stromal tumors: reappraisal of histogenesis. Am J Surg Pathol 1983;7:507-19.

2. Biasco G, Velo D, Angriman I, Astorino M, Baldan A, Baseggio M, et al. Gastrointestinal stromal tumors: report of an audit and review of the literature. Eur J Cancer Prev 2009;18:106-16.

3. Iwashita A. Clinical pathology of gastrointestinal stromal tumor (GIST) (in Japanese with English abstract). Stomach Intest 2001;36:1113-27.

4. Seya T, Tanaka N, Yokoi K, Shinji S, Oaki Y, Tajiri T. Life-threatening bleeding from gastrointestinal stromal tumor of the stomach. J Nippon Med Sch 2008;75:306-11.

5. Cheon YK, Jung IS, Cho YD, Kim JO, Lee JS, Lee MS, et al. A spontaneously ruptured gastric stromal tumor with cystic degeneration presenting as hemoperitoneum: a case report. J Korean Med Sci 2003;18:751-5.

6. De Matteo RP, Lewis JJ, Leung D, Mudan SS, Woodruff JM, Brennan MF. Two hundred gastrointestinal stromal tumors: recurrence patterns and prognostic factors for survival. Ann Surg 2000;231:51-8.

7. Nishida T, Blay JY, Hirota S, Kitagawa Y, Kang YK. The standard diagnosis, treatment, and follow-up of gastrointestinal stromal tumors based on guidelines. Gastric Cancer 2016;19:3-14.

8. Kim DJ, Lee JH, Kim W. Laparoscopic resection for 125 gastroduodenal 
submucosal tumors. Ann Surg Treat Res 2014;86:199-205.

9. Jakhetiya A, Garg PK, Prakash G, Sharma J, Pandey R, Pandey D. Targeted therapy of gastrointestinal stromal tumours. World J Gastrointest Surg
2016;8:345-52.

10. Joensuu H. Risk stratification of patients diagnosed with gastrointestinal stromal tumor. Hum Pathol 2008;39:1411-9. 